\title{
Tutoría en la Universidad: un estudio de caso en la Facultad de Educación de la Universidad de Murcia
}

\section{University Tutoring: a case study in the Faculty of Education at the University of Murcia}

\author{
Francisco Javier Pérez Cusó ${ }^{1}$ \\ javierperezcuso@um.es \\ Cristina González Lorente \\ c.gonzalezlorente@um.es \\ Natalia González Morga \\ natalia.gonzalez@um.es \\ Mirian Martínez JuÁrez \\ mmartinez@um.es \\ Universidad de Murcia, España
}

\section{Resumen:}

La tutoría está institucionalizada en todas las etapas educativas, desde Educación Infantil a la Educación Superior, siendo en el ámbito universitario donde tiene una normativa mucho más corta y reciente. A partir de la implantación del EEES, la tutoría resurge como factor de calidad del proceso educativo. A día de hoy ya no debería considerarse un aspecto novedoso hacia el que hay que tender, sino como un proceso orientador regulado y restablecido por las múltiples ventajas y beneficios que reporta a la comunidad universitaria. Este trabajo profundiza en el desarrollo de los contenidos de la tutoría y en la adecuación de su implementación desde la mirada del alumnado. La investigación parte de un enfoque metodológico cuantitativo con un diseño de investigación de corte

\begin{abstract}
:
Tutoring is institutionalized in all educative stages from childhood to higher education. However, tutoring at university level has a much shorter and newer tradition. Since the implementation of EHES, tutoring has come back as a quality factor in this educative stage, but the resurgence of university level tutoring should not be seen as a novelty but as a fundamental guiding process with many advantages and benefits to the university community. Because of that, the aim of this paper is to go into detail about the implementation and development of tutoring in this context from university students' perception. This paper is done using a quantitative methodology approach with a descriptive and transversal research design. 572 undergraduate students of Childhood Edu-
\end{abstract}

1 Dirección para correspondencia (correspondence address):

Francisco Javier Pérez Cusó. Departamento de Métodos de Investigación y Diagnóstico en Educación. Facultad de Educación. Universidad de Murcia. Campus de Espinardo, s/n. 30100 Murcia (España) 
Tutoría en la Universidad: un estudio de caso en la Facultad de Educación de la Universidad de Murcia

Francisco Javier Pérez Cusó, Cristina González lorente, Natalia González Morga y Mirian MARTíNEZ JUÁREZ

descriptivo y transversal. Los participantes son una muestra representativa de 572 estudiantes de la Facultad de Educación de la Universidad de Murcia, pertenecientes a los Grados en Educación Infantil, Educación Primaria, Educación Social y Pedagogía, los cuales aportan su percepción a partir de un cuestionario diseñado ad hoc, basado en un estudio anterior. El análisis de datos se realiza a través del programa estadístico SPSS v.21, recurriendo tanto a la estadística descriptiva como inferencial. Los resultados señalan distintas líneas de actuación para ofrecer una mayor calidad al proceso educativo desde la tutoría, especialmente la necesidad de prestar una mayor atención a los procesos de diseño de los planes de acción tutorial en consonancia con otras investigaciones como las realizadas por González y León (2014) o Cano y Paula (2008).

\section{Palabras clave:}

Educación superior; tutoría universitaria; modelo integral; contenidos de la acción tutorial. cation, Primary Education, Social Education, and Pedagogy from the University of Murcia have participated. These students contribute their opinions and evaluations through an ad hoc questionnaire based on a previous research. A reliability and validation analysis is presented through an exploratory factorial analysis. The database and its analysis were undertaken with SPSS v.21 statistics program. Descriptive and inference analysis is used on purpose. The results show different ways of intervention aimed at offering a better quality for the educative process. One especially relevant establishes that more careful attention shoud be paid to the design processes of tutoring action plans, in line with the research of González and León (2014) or Cano and Paula (2008).

\section{Key words:}

Higher education; university tutoring; integral model; contents of university tutoring.

\section{Résumé:}

Tous les stades de l'éducation, depuis la maternelle jusqu'à l'enseignement supérieur sont concernés par le tutorat institutionnalisé. Spécifiquement à l'université où elle dispose d'une législation beaucoup plus courte et récente. Depuis la mise en œuvre de I'EEES, le tutorat apparaît comme un facteur de qualité du processus éducatif actuel. II ne devrait pas être considéré comme un nouvel aspect vers lequel nous devons tendre, mais comme un processus de guidage réglementé et implémenté par les nombreux avantages et bénéfices de la communauté universitaire. Cet article explore le développement du cadre éducatif ainsi que l'adéquation de sa mise en oeuvre, du point de vue des étudiants. Ce travail de recherche s'articule autour d'une approche méthodologique quantitative avec une approche descriptive et transversale. Les participants sont un échantillon représentatif de 572 étudiants de la Faculté d'Education de l'Université de Murcia, appartenant à la Licence spécialisée en petite-enfance, enseignement primaire, enseignement social et de la pédagogie. Leurs perceptions ont été recueillies à travers d'un questionnaire conçu ad hoc sur la base d'une étude précédente. L'analyse des données a été réalisée par le biais SPSS v.21, utilisant à la fois des statistiques descriptives et inférentielles. Les résultats montrent des lignes d'action distinctes pour offrir une meilleure qualité au processus éducatif de tutorat, en particulier la nécessité d'accorder une plus grande attention aux processus de conception des plans d'action de tutoriel en ligne avec d'autres études telles que celles menées par González y León (2014) ou Cano et Paula (2008).

\section{Mots clés:}

L'enseignement supérieur; tutorat académique; modèle global; contenus de l'action tutoriel.

Fecha de recepción: 24-2-2017

Fecha de aceptación: 10-3-2017 


\section{Introducción}

La tutoría universitaria es uno de los elementos clave del proceso de integración de las universidades en el Espacio Europeo de Educación Superior (en adelante EEES). Docentes, estudiantes y analistas la asumen como un factor decisivo en el desarrollo de una Educación Superior de calidad, puesto que está destinada a favorecer el desarrollo integral del estudiante tanto en el progreso académico como en el personal y profesional (Martínez, Pérez y Martínez, en prensa; Rodríguez Espinar, 2001)

Al igual que ocurre en otras etapas educativas, en el contexto universitario no es fácil modificar modos de hacer, estructuras, ideas previas, tradiciones, etc., en definitiva, modificar la propia cultura institucional, más aún cuando estos cambios se trasladan al desarrollo de la tutoría en la Educación Superior que, más allá de experiencias e iniciativas puntuales, no goza de un largo recorrido y reconocimiento.

El interés e importancia de este tema queda asegurado cuando se revisan los diferentes estudios e investigaciones que, a lo largo de la última década, se han realizado en torno a este tópico de estudio. En ellos se señalan algunos déficits y áreas de mejora como, por ejemplo, el estudio de Manzano, Martín, Sánchez, Rísquez y Suárez (2012), en el que se destacan algunos de los problemas en el desarrollo de la tutoría entre iguales; Torrecilla, Rodríguez, Herrera y Martín (2013) se centran en las necesidades y percepción de los alumnos de reciente ingreso en relación a la tutoría, o Martínez, Pérez y Martínez (2016), que debaten sobre el uso de las TIC en la tutoría. Estos y otros estudios muestran el interés de investigadores de diferentes universidades por esta temática en la institución universitaria, exponiendo la variedad de ámbitos, modalidades y figuras presentes en el propio concepto de tutoría.

Desde la tutoría universitaria se plantean diferentes modalidades: académica, personal, de titulación o carrera, entre iguales, de servicio, de prácticas, de investigación (Lobato y Guerra, 2016). Estas deben dar respuesta a los tres grandes ámbitos que conforman la visión o concepción de una tutoría universitaria integral: ámbito académico, ámbito profesional y ámbito personal, extendiéndose más allá de la mera supervisión de trabajos de asignatura y resolución de dudas previas a la realización de exámenes u otras pruebas finales.

Con la atención puesta en el epicentro de esta acción, el desarroIlo integral del estudiante, la tutoría en el contexto universitario puede 
Tutoría en la Universidad: un estudio de caso en la Facultad de Educación de la Universidad de Murcia

Francisco Javier Pérez Cusó, Cristina González lorente, Natalia González Morga y Mirian MARTíNEZ JUÁREZ

convertirse en uno de los elementos diferenciadores que sustenten la calidad de la formación (Álvarez y Álvarez, 2015; Cano, 2009; Lobato y Guerra, 2014; Martínez, Pérez y Martínez, 2014). A través de sus diferentes modalidades y ámbitos se debe trabajar y dar respuesta a distintas necesidades de orientación, apoyo y acompañamiento del alumnado, prestando atención a:

- La adaptación e integración al contexto universitario. Se trata de uno de los aspectos clave de la tutoría, especialmente al ingreso en la institución, creando un proceso de acompañamiento a los estudiantes nóveles al inicio de su transición universitaria. Nuevas formas de enseñar y aprender, nuevos espacios y modos de hacer, nuevos contenidos, compañeros y docentes, suponen una adaptación en la que se puede, y debe, actuar desde la tutoría (Álvarez Pérez, 2012a; García, Carpintero, Biencinto y Núñez, 2014; Torrecilla, Rodríguez, Herrera y Martín, 2013). Un adecuado apoyo y acompañamiento puede reducir el abandono y fracaso académico (Da Re, Álvarez, y Clerici, 2015; Esteban, Bernardo y RodríguezMuñiz, 2016; González, Álvarez, Cabrera y Bethencourt, 2007).

- El desarrollo académico. La tutoría debe ir más allá de asesorar sobre contenidos de aprendizaje y evaluación; le corresponde favorecer el desarrollo de estrategias de aprendizaje, la planificación y gestión del tiempo de trabajo y estudio y potenciar la reflexión y la toma de decisiones en torno al itinerario académico personalizado: elección de asignaturas optativas, prácticas curriculares, etc. Se convierte en una acción clave y necesaria para que los estudiantes realicen un aprendizaje integrado, afrontando el riesgo de parcelación y balcanización, propio de la enseñanza universitaria.

- La definición del perfil profesional. La tutoría está llamada a tener un papel relevante en la definición del perfil profesional de cada estudiante, facilitando la elaboración del proyecto profesional y de vida. La tutoría promueve una toma de decisiones reflexiva, crítica, responsable y adaptada a la situación particular de cada estudiante, con el objetivo de escoger opciones encaminadas a reconducir en todo momento su carrera profesional.

- El desarrollo de competencias profesionales. Como apoyo al proceso formativo, desde la tutoría se potencia y dinamiza el desarrollo de competencias que van a ser clave en el futuro desarrollo profesional del alumnado y que, por tanto, van a facilitar su futura 
inserción socio-laboral (Cano, 2009; Sobrado, 2008), facilitando la transición a la vida activa y la inserción socio-profesional.

- La transición al mercado laboral. Especialmente en los últimos cursos de Grado, pero también en la formación de postgrado, la tutoría debe actuar en la compleja transición entre la universidad y el mundo del trabajo, facilitando los procesos de búsqueda de empleo y de inserción socio-laboral. Algunos estudios (Gil-Albarova, Martínez Odriá, Tunnicliffe y Miguel Moneo, 2013) señalan que esta es la principal demanda del alumnado a la tutoría universitaria.

- El desarrollo personal. Gastón y Rekalde (2016) inciden en que el ámbito personal de la tutoría es uno de los menos desarrollados, incluso el profesorado participante en algunos estudios muestra sus reticencias a trabajar determinados aspectos de la dimensión más personal. Las posturas frente a esta cuestión se posicionan en un continuo entre dos polos: por un lado, aquellos que destierran la respuesta a este tipo de necesidades a otros ámbitos y profesionales y, por otro, aquellos que opinan que no es posible disociar lo personal de lo académico (Haya, Calvo y Rodríguez-Hoyos, 2013). Pese al escaso consenso que genera este ámbito, Sobrado (2008) y López Gómez (2015) recogen algunos elementos que desde la tutoría se deben trabajar en el ámbito del desarrollo personal: ayuda en los procesos madurativos, favorece la actitud crítica, fomenta el desarrollo de la autonomía personal, el proceso de toma de decisiones, el conocimiento de uno mismo, autoestima etc.

- Resolución de conflictos. A lo largo del curso académico pueden surgir diferentes conflictos, tanto dentro del grupo-clase como en la relación con el profesorado, y desde la tutoría se puede asumir una función de mediación que posibilite el entendimiento y la resolución constructiva de los conflictos que puedan acontecer, de tal manera que no sólo se puedan solucionar, sino que su solución pueda ser parte de un proceso de aprendizaje del alumnado y del centro universitario.

Como puede apreciarse, las necesidades sobre las que ha de actuar la tutoría la convierten en una acción nuclear en nuestras universidades, si verdaderamente desean ofrecer una formación integral y de calidad, que atienda a las peculiaridades de un alumnado mucho más heterogéneo y diverso que décadas atrás. 
Tutoría en la Universidad: un estudio de caso en la Facultad de Educación de la Universidad de Murcia

Francisco Javier Pérez Cusó, Cristina González lorente, Natalia González Morga y Mirian MARTíNeZ JuÁreZ

Su necesidad y relevancia es indiscutible, más allá de aceptar la tutoría universitaria como un proceso inherente al quehacer docente y al tutor como una figura de referencia para el alumnado. Es preciso indagar en cuáles son los contenidos sobre los que debe profundizar y cuáles son los que el alumnado plantea con mayor demanda.

Esta investigación contribuye al avance de la acción tutorial en el contexto universitario con un doble propósito: valorar con mayor pertinencia los requerimientos de orientación del alumnado y responder a los mismos de forma precisa; para ello se concretan los siguientes objetivos: 1) conocer y analizar la percepción del alumnado en relación al desarrollo de los contenidos planteados desde la tutoría en la Facultad de Educación de la Universidad de Murcia, 2) valorar la adecuación de los mismos y 3) plantear propuestas de mejora.

\section{Método}

Para alcanzar los objetivos propuestos se emplea un diseño no experimental de tipo descriptivo y transversal, a través de una metodología tipo encuesta. La información se recoge a través de un cuestionario diseñado ad hoc, formado por diferentes escalas y una pregunta abierta final en la que se solicita a los participantes ofrecer propuestas de mejora. Se presentan los resultados relativos a la escala de percepción del desarrollo de los contenidos de la tutoría, cuyas respuestas a cada ítem se gradúan de 1 a 10 (siendo $1=$ nada y $10=$ mucho).

Los participantes son estudiantes de los cuatro títulos de Grado de la Facultad de Educación de la Universidad de Murcia. Para obtener una información más fiable se accede al alumnado de segundo, tercer y cuarto curso matriculados en 2016-2017, prescindiendo del alumnado de primer curso, dada su escasa toma de contacto con la tutoría universitaria. La recogida de información se realiza en horas de clase, solicitando el permiso y colaboración de los tutores de los grupos o de otros profesores, en el caso de que el tutor no imparta docencia en el cuatrimestre en el que se aplica. Son los miembros del grupo de investigación quienes se encargan de organizar y supervisar la cumplimentación de los cuestionarios, garantizando su correcta aplicación.

La muestra recogida es de 572 participantes, lo que asegura un 97\% de nivel de confianza y un error menor del $4 \%$, teniendo en cuenta una 
población total de $\mathrm{N}=3823$ estudiantes matriculados en estudios de Grado en la Facultad de Educación de la Universidad de Murcia, así como la mayor variabilidad posible (50\%). La distribución de los participantes por titulaciones y sexo se muestra en la tabla 1.

Tabla 1. Participantes por titulación y sexo.

\begin{tabular}{lccccc}
\hline & Hombres $(\mathrm{n})$ & $\%$ & Mujeres $(\mathrm{n})$ & $\%$ & Total $(\mathrm{n})$ \\
\hline Educación Infantil & 1 & $0.88 \%$ & 112 & $99.12 \%$ & 113 \\
Educación Primaria & 76 & $28.46 \%$ & 191 & $71.54 \%$ & 267 \\
Educación Social & 13 & $13.83 \%$ & 81 & $86.17 \%$ & 94 \\
Pedagogía & 14 & $14.29 \%$ & 84 & $85.71 \%$ & 98 \\
\hline Total & 104 & $18.18 \%$ & 468 & $81.82 \%$ & 572 \\
\hline
\end{tabular}

El $33.22 \%$ de los participantes están matriculados en segundo curso, mientras que los matriculados en tercer y cuarto curso conforman un $27.45 \%$ y un $39.34 \%$ de la muestra, respectivamente. La edad media de los participantes es de 21.82 años (DT=3.51). Es importante señalar que un $26.85 \%$ de la muestra compatibiliza los estudios con algún trabajo remunerado.

Tras la aplicación del instrumento a los participantes se procede a incluir la información en una base de datos y realizar su posterior análisis con el programa estadístico SPSS v.21, efectuando el pertinente análisis de fiabilidad, mediante el alfa de Cronbach, y de validez, mediante Análisis Factorial Exploratorio (en adelante, AFE). Para la comparación entre grupos se emplean los estadísticos no paramétricos $U$ de Mann Whitney, prueba de Kruskal-Wallis y prueba de Friedman.

\section{Resultados}

En primer lugar se presenta el análisis factorial y de fiabilidad de la escala de percepción del desarrollo de los contenidos de la tutoría, así como las subescalas basadas en dicho análisis factorial. La medida de adecuación muestral $(\mathrm{KMO}=.959)$ y la prueba de esfericidad de Bartlett (Chi-cuadrado=10845.338, gl=210, sig=.000) muestran la idoneidad para aplicar el AFE. Dicho análisis se realiza mediante extracción de componentes principales y rotación Varimax con Káiser, arrojando una composición de tres factores que conjuntamente explican un $72.17 \%$ de 
Tutoría en la Universidad: un estudio de caso en la Facultad de Educación de la Universidad de Murcia

Francisco Javier Pérez Cusó, Cristina González lorente, Natalia González Morga y Mirian MARTíNEZ JUÁREZ

la varianza. En la tabla 2 se muestra la matriz de componentes rotados, ordenados según sus pesos factoriales.

Tabla 2. Matriz de componentes rotados.

\begin{tabular}{|c|c|c|c|}
\hline & 1 & 2 & 3 \\
\hline Ítem 13 & .749 & & \\
\hline Ítem 11 & .725 & & \\
\hline Ítem 2 & .684 & & \\
\hline Ítem 12 & .682 & & .415 \\
\hline Ítem 10 & .682 & & .452 \\
\hline Ítem 15 & .634 & .457 & \\
\hline Ítem 14 & .632 & .477 & \\
\hline Ítem 17 & .582 & .437 & \\
\hline Ítem 3 & .565 & .493 & \\
\hline Ítem 20 & & .784 & .441 \\
\hline Ítem 19 & & .766 & \\
\hline Ítem 18 & & .761 & \\
\hline Ítem 21 & & .745 & .461 \\
\hline Ítem 16 & .483 & .586 & \\
\hline Ítem 4 & .440 & .555 & \\
\hline Ítem 1 & .461 & .531 & \\
\hline Ítem 6 & & & .809 \\
\hline Ítem 8 & & & .798 \\
\hline Ítem 5 & & & .772 \\
\hline Ítem 7 & & & .768 \\
\hline Ítem 9 & & .424 & .756 \\
\hline
\end{tabular}

Se incluyen los pesos factoriales superiores a .4, marcándose en negrita los que se han considerado para la inclusión final en las subescalas basadas en el análisis factorial. En todos los casos se incluyen los ítems con peso factorial más alto, exceptuando el ítem $n^{\circ} 1$ que se incluye en la primera subescala debido a que presenta pesos factoriales muy parecidos en el primer y segundo factor, pero, desde el punto de vista del contenido, resulta más coherente en la primera de las escalas.

El análisis de fiabilidad arroja un alfa de Cronbach muy alto, tanto para la escala global $(\alpha=.964)$ como para la primera de las subescalas, relativa a la percepción del desarrollo de la tutoría académica $(\alpha=.928)$, en la que se incluyen 10 ítems; la segunda subescala, referida a percep- 
ción del desarrollo de la tutoría profesional $(\alpha=.909)$, compuesta por 6 ítems, y la tercera, concerniente a la percepción del desarrollo de la tutoría personal, compuesta por 5 elementos y con un alfa igual a .967.

En la tabla 3 se presentan los ítems correspondientes a la subescala de percepción del desarrollo de la tutoría académica, objeto de este estudio, mostrándose la media y desviación típica de cada contenido.

Tabla 3. Elementos que componen la subescala de percepción del desarrollo de la tutoría académica.

\begin{tabular}{|c|c|c|c|}
\hline Ítem & Descripción & Media $(\bar{x})$ & Desv. Típica $(\sigma)$ \\
\hline 13 & $\begin{array}{l}\text { Toma de decisiones grupales (elección de } \\
\text { delegado/a, comunicación con profesorado, } \\
\text { etc.) }\end{array}$ & 5.91 & 3.047 \\
\hline 11 & $\begin{array}{l}\text { Trabajo en equipo (colaborativo y coopera- } \\
\text { tivo) }\end{array}$ & 5.19 & 2.990 \\
\hline 2 & $\begin{array}{l}\text { Plan de estudios (ordenación, asignaturas, } \\
\text { exámenes, prácticas, TFG/TFM, etc.) }\end{array}$ & 4.83 & 2.730 \\
\hline 12 & $\begin{array}{l}\text { Resolución de conflictos surgidos en el gru- } \\
\text { po-clase }\end{array}$ & 4.45 & 2.955 \\
\hline 10 & Conocimiento del grupo-clase & 4.45 & 2.800 \\
\hline 15 & Planificación y gestión del tiempo & 3.84 & 2.634 \\
\hline 14 & $\begin{array}{l}\text { Estrategias de aprendizaje (para facilitar la } \\
\text { gestión, organización, interpretación y evo- } \\
\text { cación de la información) }\end{array}$ & 3.99 & 2.659 \\
\hline 17 & Trabajos académicos y científicos & 4.01 & 2.675 \\
\hline 3 & Tus derechos y deberes como alumno/a & 4.03 & 2.692 \\
\hline 1 & Estructura y organización del centro & 3.57 & 2.424 \\
\hline
\end{tabular}

Como puede apreciarse, en esta subescala se agrupan los ítems relacionados con el acompañamiento en los procesos de enseñanza-aprendizaje, el desarrollo académico y la relación con el grupo-clase. En términos generales se puede apreciar que sólo dos de los ítems superan el cinco, destacando especialmente la puntuación media del ítem $\mathrm{n}^{\circ} 13$, "Toma de decisiones grupales (elección de delegado, comunicación con el profesorado, etc.)", contenido que está muy reglamentado, por lo que es lógico que sea el que obtiene una mayor puntuación.

Los ítems que obtienen una puntuación inferior son los referidos a la información sobre la estructura y organización del centro $(\bar{x}=3.57)$, a la planificación y gestión del tiempo $(\bar{x}=3.84)$ o los relacionados con las estrategias de aprendizaje $(\bar{x}=3.99)$. 
Tutoría en la Universidad: un estudio de caso en la Facultad de Educación de la Universidad de Murcia

Francisco Javier Pérez Cusó, Cristina González lorente, Natalia González Morga y Mirian MARTíNeZ JuÁreZ

Por su parte, la subescala de percepción del desarrollo de la tutoría profesional queda formada por 6 ítems que se presentan en la tabla 4, junto con la media y la desviación típica de cada uno de ellos.

Tabla 4. Elementos que componen la subescala de percepción del desarrollo de la tutoría profesional.

\begin{tabular}{|c|c|c|c|}
\hline Ítem & Descripción & Media $(\bar{x})$ & Desv. Típica $(\sigma)$ \\
\hline 20 & Proceso de búsqueda activa de empleo & 2.61 & 2.295 \\
\hline 19 & $\begin{array}{l}\text { Diseño de tu Proyecto Profesional y de } \\
\text { Vida }\end{array}$ & 3.02 & 2.538 \\
\hline 18 & Mercado laboral & 3.00 & 2.418 \\
\hline 21 & $\begin{array}{l}\text { Desarrollo de competencias para la em- } \\
\text { pleabilidad (inserción socio-laboral) } \\
\text { ltinerario formativo y curricular (elección }\end{array}$ & 2.77 & 2.394 \\
\hline 16 & $\begin{array}{l}\text { de asignaturas optativas, prácticas curricu- } \\
\text { lares, temática de TFG/TFM, etc.) }\end{array}$ & 3.82 & 2.664 \\
\hline 4 & $\begin{array}{l}\text { Actividades de interés en las que puedes } \\
\text { participar (cursos, programas- de intercam- } \\
\text { bio, de prácticas-, voluntariado, talleres, } \\
\text { conferencias, etc.) }\end{array}$ & 4.11 & 2.776 \\
\hline
\end{tabular}

En esta subescala se agrupan ítems relacionados con tres contenidos de la tutoría en el ámbito profesional: la adquisición de competencias profesionales, el diseño de un itinerario profesional y el proceso de inserción socio-laboral. Puede apreciarse que los ítems referidos a éste último contenido son los que menores puntuaciones medias obtienen (ítem 20, $\bar{x}=2.61$; ítem $18, \bar{x}=3.00$ ), mientras que los ítems que obtienen mayores puntuaciones, aunque siempre por debajo de 5, son los referidos al acompañamiento en el diseño de un itinerario individualizado, como el ítem $\mathrm{n}^{\circ} 4$, "Actividades de interés en las que puedes participar" $(\bar{x}=4.11)$, o el $\mathrm{n}^{\circ} 16$, "Itinerario formativo y curricular" $(\bar{x}=3.82)$.

Por último se presentan los elementos que componen la subescala de percepción del desarrollo de la tutoría personal, compuesta por un total de 5 elementos relacionados con el conocimiento de sí mismo, la clarificación de intereses, la toma de decisiones e, incluso, el manejo de situaciones de estrés y ansiedad. 
Tabla 5. Elementos que componen la subescala de percepción del desarrollo de la tutoría personal.

\begin{tabular}{clcc}
\hline Ítem & Descripción & Media $(\bar{x})$ & Desv. Típica $(\sigma)$ \\
\hline \multirow{2}{*}{6} & Identificación de tus fortalezas y debilida- & 3.28 & 2.644 \\
& des & 3.35 & 2.684 \\
8 & Proceso de toma de decisiones personal & 3.36 & 2.654 \\
5 & Conocimiento de ti mismo y del entorno & 3.46 & 2.663 \\
7 & Clarificación de tus intereses y expectativas & 36 \\
9 & Control de la adversidad (estrés y ansiedad) & 2.97 & 2.568 \\
\hline
\end{tabular}

Puede apreciarse en la tabla 5 que todos los elementos obtienen unas puntuaciones muy bajas, destacando especialmente el ítem $n^{\circ} 9$, referido al control del estrés y la ansiedad, que arroja una media de 2.97.

Con estas tres subescalas se puede valorar la profundidad del desarrollo de los diferentes ámbitos de la tutoría (académica, profesional y personal), para lo que se presentan comparaciones entre grupos a través de pruebas no paramétricas. En primer lugar, se presenta la comparación entre las tres subescalas mediante la prueba de Friedman, cuyos resultados constatan que las diferencias entre las tres escalas son significativas (chi-cuadrado $=342,520 ; \mathrm{gl}=2$; sig. $=.000$ ), tal y como se muestra en la tabla 6.

Tabla 6. Estadísticos subescalas.

\begin{tabular}{lccc}
\hline & Media $(\bar{x})$ & $\begin{array}{c}\text { Desviación } \\
\text { típica }(\sigma)\end{array}$ & $\begin{array}{c}\text { Rango } \\
\text { promedio }\end{array}$ \\
\hline $\begin{array}{l}\text { Subescala de percepción de desarrollo } \\
\text { tutoría académica }\end{array}$ & 4.4319 & 2.15653 & 2.60 \\
$\begin{array}{l}\text { Subescala de percepción de desarrollo } \\
\text { tutoría profesional }\end{array}$ & 3.2324 & 2.09978 & 1.74 \\
$\begin{array}{l}\text { Subescala de percepción de desarrollo } \\
\text { tutoría personal }\end{array}$ & 3.2920 & 2.48762 & 1.66 \\
\hline
\end{tabular}

Se observa que el alumnado percibe que la modalidad de tutoría más desarrollada es la académica, seguida de la tutoría personal y profesional.

En la comparación entre las cuatro titulaciones participantes en el estudio, la prueba de Kruskal-Wallis muestra diferencias significativas tanto en la escala global (chi-cuadrado=21.995; gl=3; sig.=.000) como en la subescala referida a la tutoría académica (chi-cuadrado=29.924; gl=3; 
Tutoría en la Universidad: un estudio de caso en la Facultad de Educación de la Universidad de Murcia

Francisco Javier Pérez Cusó, Cristina González lorente, Natalia González Morga y Mirian MARTíNEZ JUÁREZ

sig.=.000) y en la relativa a la tutoría personal (chi-cuadrado $=16.038$; $\mathrm{gl}=3$; sig.=.001), no así con la tutoría profesional (chi-cuadrado=5.910; $\mathrm{gl}=3$; sig.=.116). En la figura 1 se pueden apreciar los datos de las subescalas agrupadas por titulaciones.

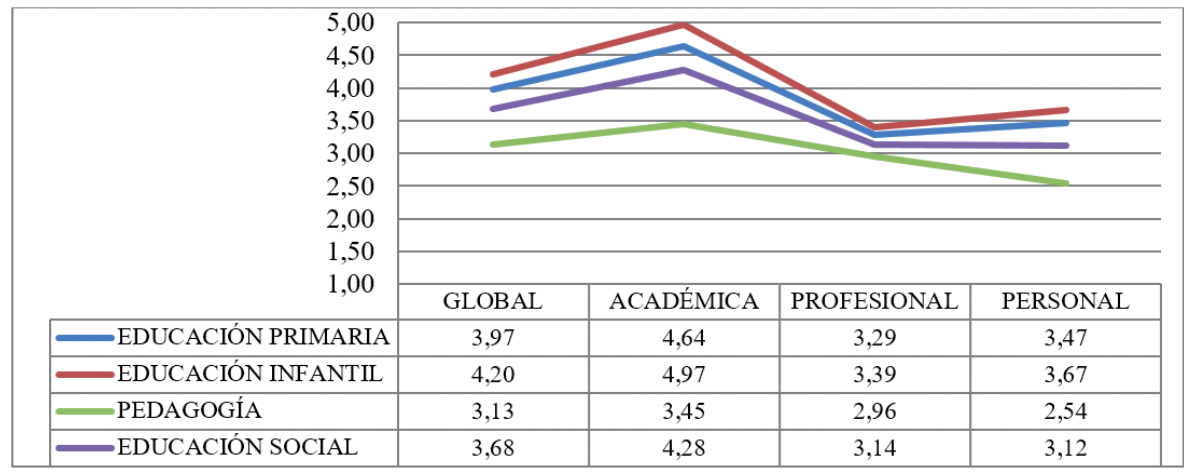

Figura 1. Puntuaciones de escala y subescalas según titulación.

Se observa que tanto la escala global como las tres subescalas muestran un comportamiento similar en las cuatro titulaciones, siendo superior la percepción de desarrollo de la tutoría académica entre los estudiantes de las cuatro titulaciones.

También resulta interesante analizar los resultados en las diferentes escalas según el curso en que están matriculados los participantes. La prueba de Friedman muestra diferencias significativas $(p>.05)$ en la escala global (chi-cuadrado=17.037; gl=2; sig.=.000) y en las referidas al desarrollo académico (chi-cuadrado=19.748; gl=2; sig.=.000), al desarroIlo profesional (chi-cuadrado=9.977; gl=2; sig.=.007) y al desarrollo de la tutoría personal (chi-cuadrado=6.257; gl=2; sig.=.044). Los resultados se representan en la figura 2 , mostrando la tendencia de los estudiantes de cursos superiores a obtener una mayor puntuación tanto en la escala global como en las tres subescalas. 


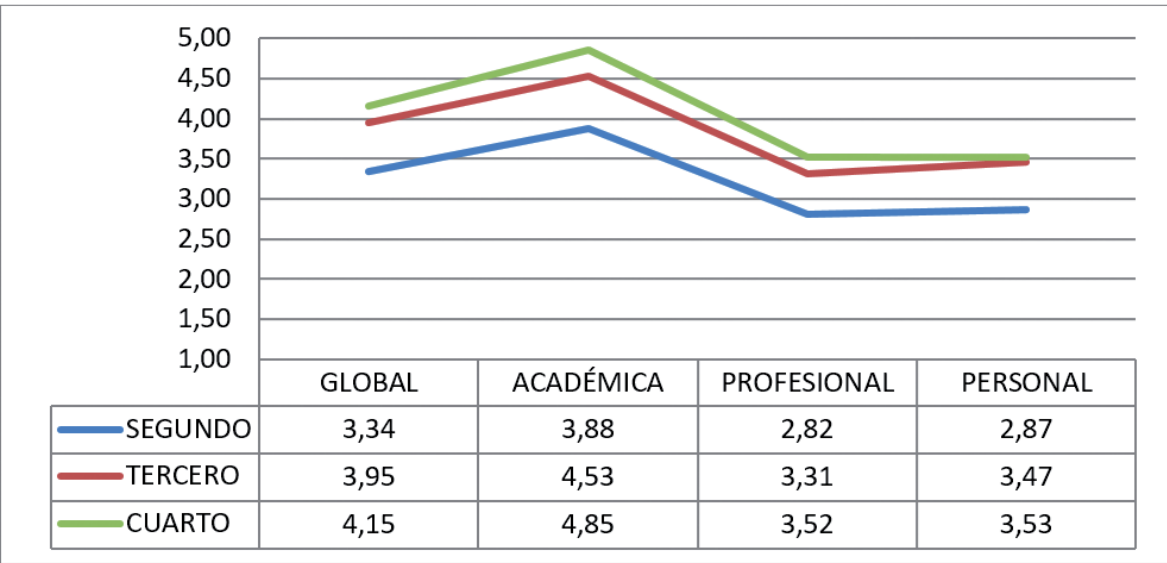

Figura 2. Puntuaciones de escala y subescalas según curso.

A la hora de comparar según el sexo de los participantes, la prueba $U$ de Mann-Whitney no muestra diferencias significativas $(p<.05)$, salvo en la escala referida al desarrollo de la tutoría en el ámbito de desarrollo profesional, arrojando los hombres $(\bar{x}=3.58)$ una media algo superior a las mujeres $(\bar{x}=3.15)$. Asimismo se compara la muestra de estudiantes que trabajan con aquellos que no realizan ninguna labor remunerada, no hallándose diferencias significativas $(p<.05)$, ni en la escala global ni en ninguna de las tres subescalas, a través de la prueba $U$ de Mann-Whitney

Por último, se presenta la comparación por grupos de edad. En esta ocasión sí existen diferencias significativas $(p<.05)$ tanto en la escala global como en las tres subescalas entre los alumnos más jóvenes $(<25$ años) y los más veteranos (>25 años), utilizando la misma prueba.

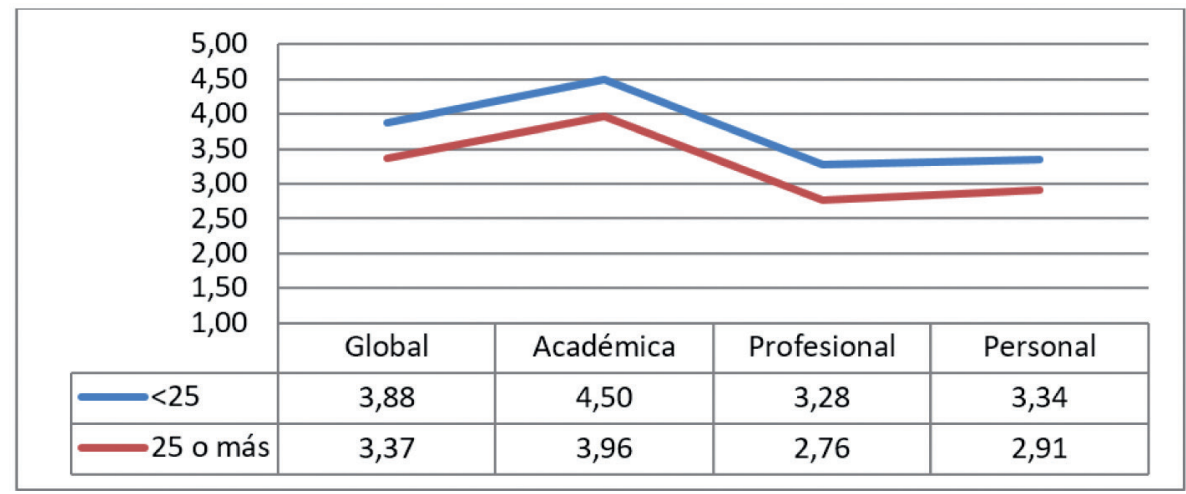

Figura 3. Puntuaciones escala y subescalas según edad de los participantes. 
Tutoría en la Universidad: un estudio de caso en la Facultad de Educación de la Universidad de Murcia

Francisco Javier Pérez Cusó, Cristina González lorente, Natalia González Morga y Mirian MARTíNeZ JuÁreZ

Como se aprecia en la figura 3, en todas las escalas analizadas son los estudiantes de menor edad quienes muestran una mayor puntuación, lo que puede indicar una mejor adecuación de la tutoría a sus necesidades. Por su parte, el alumnado mayor de 25 años se muestra más crítico con el desarrollo de los tres ámbitos de la tutoría.

\section{Discusión y conclusiones}

A partir de la experiencia acumulada en estudios realizados anteriormente, del diseño ad hoc de este cuestionario y su posterior validación de contenido a través del Análisis Factorial Exploratorio, se dispone de un instrumento válido y fiable para valorar el desarrollo de contenidos en la tutoría universitaria, relacionados con los ámbitos académico, profesional y personal.

La comparación de las tres subescalas pone de relieve que el alumnado percibe un escaso desarrollo de la tutoría en general, mostrando en todos los casos puntuaciones muy bajas. Se constata que es la tutoría académica, con elementos como la toma de decisiones grupales, el trabajo en equipo, estrategias de aprendizaje, etc., la que presenta una mayor valoración en relación a los ámbitos personal y profesional. Estos datos coinciden con diferentes estudios, como los desarrollados por Gastón y Rekalde (2016), Martínez, Martínez y Pérez (2014) y Amor (2016), en los que se subraya que la dimensión o área de trabajo de la tutoría universitaria más asimilada por profesores y estudiantes es la académica, quedando el resto de contenidos y ámbitos de la tutoría relegados a un segundo plano. Torrecilla, Rodríguez, Herrera y Martín (2013) señalan que, a pesar de ser la tutoría en el ámbito académico la que tradicionalmente se implementa más, es el tratamiento de temas relacionados con el desarrollo personal el que tiene un alto impacto en la satisfacción global del alumnado con la misma. No obstante, en otros estudios, como el de López Gómez (2015), se plantea si es adecuado el trabajo en este ámbito dentro del contexto universitario, manifestando que es el propio profesorado el que se muestra reticente.

Otra importante conclusión es la relativa a las diferentes percepciones que tiene el alumnado perteneciente a las cuatro titulaciones estudiadas en relación al desarrollo de los contenidos de la tutoría, hallándose diferencias significativas. Estas diferencias parecen lógicas, puesto 
que los contenidos impartidos en cada titulación deben responder a las necesidades de orientación del alumnado, aunque partan de un tronco común. Es por ello que los planes de acción tutorial de cada Facultad deberían plantearse a partir de las demandas identificadas en cada centro, adaptándose después a las particularidades de cada titulación y, por supuesto, de cada curso.

Igualmente, el curso académico en el que se encuentran los participantes también arroja diferencias significativas en la percepción del desarrollo de los contenidos de la tutoría universitaria. En esta ocasión es el alumnado de cursos superiores el que señala un mayor desarrollo de la tutoría en los tres ámbitos estudiados. Otros trabajos, como el de Martínez, Martínez y Pérez (2014), también señalan diferencias en la percepción del alumnado de distinto curso en cuanto al desarrollo y la utilización de la tutoría. Por su parte, Lobato y Guerra (2014) advierten que, tanto en España como en una parte importante de países del contexto europeo, el mayor esfuerzo de los planes de acción tutorial suele centrarse en la transición a los estudios universitarios (planes de incorporación, módulos cero, mentorización, etc.), dada las concretas necesidades de los estudiantes recién incorporados. No obstante, es preciso no olvidar que los estudiantes de cursos superiores van a presentar otras necesidades en el progreso de su carrera universitaria, principalmente enfocadas a los procesos de transición al mundo del trabajo (Campoy y Pantoja, 2000), pero también ligadas a la elección de formación de postgrado, de prácticas curriculares y extracurriculares, de realización de Trabajos Fin de Grado, etc.

Respecto a la comparación por grupos de edad aparecen diferencias en las tres subescalas, revelándose que los estudiantes de mayor edad son más críticos con la percepción del desarrollo de la tutoría en sus diferentes ámbitos. Otros estudios también encuentran diferencias según la edad del alumnado, como el desarrollado por López Gómez (2015) que indica que los estudiantes de menor edad valoran más la tutoría dentro del ámbito personal, mientras que los estudiantes de mayor edad destacan la tutoría relacionada con los procesos de inserción y desarroIlo profesional. Por su parte, el reciente trabajo de Amor (2016) expone que el alumnado de más edad tiende a acudir con mayor frecuencia a las tutorías, mientras que el realizado por Martínez, Pérez y Martínez (2014) muestra una menor satisfacción con la tutoría en los estudiantes de más edad. 
Tutoría en la Universidad: un estudio de caso en la Facultad de Educación de la Universidad de Murcia

Francisco Javier Pérez Cusó, Cristina González lorente, Natalia González Morga y Mirian MARTíNeZ JuÁreZ

Solaguren-Bescoa y Moreno (2016) señalan que en nuestro sistema universitario existe una escasa cultura en relación a la tutoría universitaria, como demuestra la presente investigación con el escaso desarrollo de los contenidos de la tutoría presentados en las diferentes subescalas (siendo los ítems de la subescala de percepción del desarrollo de la tutoría académica los que obtienen una media más elevada, sin superar en su mayoría el 5).

Se concluye también que es preciso implementar los tópicos clave de la tutoría, expuestos en la escala de percepción del desarrollo de los contenidos de la tutoría del presente trabajo y que parten de un concepto globalizador e integral de tutoría, contemplándola como un elemento de calidad universitaria, tal y como señalan diversos autores (Álvarez y Álvarez, 2015; Lobato y Guerra, 2014; Martínez, Pérez y Martínez, 2014). A partir de este modelo integral confluyen los diferentes ámbitos o dimensiones en los que debe incidir la tutoría -académico, personal y profesional-, así como las distintas modalidades y figuras necesarias para su desarrollo y mejora.

En este sentido, se considera la tutoría universitaria como un proceso clave de la Educación Superior, no como una actividad paralela que desarrolla parte del profesorado desde una implicación personal que no es ni exigida ni reconocida adecuadamente, como bien señala Cano (2008, p. 128):

La Tutoría, pues, aparece así como uno de los mimbres a tener en cuenta en el tejido de la nueva Universidad; como un elemento inherente y consustancial a la función docente, como un factor de concreción práctica de la actividad orientadora de carácter integral; en definitiva, como un elemento de calidad y excelencia de la Educación Superior.

A partir de los resultados de este estudio se evidencia la necesidad de realizar una planificación en mayor profundidad de la tutoría, poniendo en valor la importancia de los planes de acción tutorial y el trabajo conjunto que sobre los mismos han de realizar tanto las autoridades académicas como los docentes, e incluso el alumnado (Sobrado, 2008), teniendo en cuenta los siguientes aspectos:

- En la tutoría se deben planificar contenidos de trabajo más allá de los puramente burocráticos, como la elección de delegado/a de clase, diseñando tópicos de trabajo dentro de sus diferentes ámbitos. 
- Las necesidades de orientación y tutoría del alumnado, a medida que avanza en sus estudios, son diferentes, por lo que en el diseño y planificación de los planes de acción tutorial debe tenerse en cuenta la especificación de diferentes objetivos acordes al momento en que se sitúa el estudiante y sus necesidades.

- En una Facultad con titulaciones diversas debe tenerse en cuenta que las necesidades de concreción de la carrera profesional, e incluso en el propio acompañamiento académico, pueden ser diferentes, por tanto deben considerarse en la planificación y puesta en práctica de la acción tutorial. En este sentido, Álvarez (2012b) advierte que es necesario vincular la tutoría de carrera con cada titulación de modo que se favorezca la adquisición de competencias relevantes para cada una.

- Frente a un alumnado uniforme, característico de la universidad de tiempos pasados, la actual universidad se caracteriza por la diversidad de sus estudiantes según diferentes variables (sexo, edad, obligaciones laborales y familiares, motivaciones e intereses, necesidades educativas específicas, cultura, etc.) que se deben atender en los procesos de tutorización y, por tanto, en el diseño de los planes de acción tutorial.

Para poder cumplir con estas exigencias es necesario asumir la tutoría universitaria como un proceso integrado inexcusablemente en la práctica docente y organizativa de los centros universitarios, que deben hacer un esfuerzo en su planificación. Ahora bien, es preciso que dicha planificación huya de los meros requisitos formales y burocráticos y profundice en las verdaderas necesidades de orientación, apoyo y acompañamiento del alumnado. Desde esa adecuada planificación se podrá ofrecer una respuesta comprensiva e integral a los estudiantes, potenciando y fortaleciendo su formación y desarrollo como futuros profesionales.

La actitud y compromiso de la comunidad universitaria mejorará si se consigue potenciar un modelo integral de tutoría que dé una respuesta globalizada e integradora a todas las necesidades de orientación del alumnado, a través de un sistema holístico de orientación que sea accesible y cercano a todos, enmarcado en un sistema de garantía de calidad que asegure su control y mejora permanente y continua (Echeverría y Martínez Clares, 2015, p. 17). 
Tutoría en la Universidad: un estudio de caso en la Facultad de Educación de la Universidad de Murcia

Francisco Javier Pérez Cusó, Cristina González lorente, Natalia González Morga y Mirian MARTíNEZ JUÁREZ

\section{Referencias bibliográficas}

Amor, M. I. (2016). Evaluación de la Orientación y la Tutoría en la Facultad de Ciencias de la Educación de la Universidad de Córdoba. Educatio Siglo XXI, 34(1), 93-112.

Álvarez, M., \& Álvarez, J. (2015). La tutoría universitaria: del modelo actual a un modelo integral. Revista Electrónica Interuniversitaria de Formación Del Profesorado, 18(2), 125-142. Recuperado de http://dialnet.unirioja.es/servlet/articulo?codigo=5097 275 \&orden $=0$ \&info $=$ link

Álvarez Pérez, P. (2012a). Los planes de tutoría de carrera: una estrategia para la orientación al estudiante en el marco del EEES. Educar, 48(2), 247-266.

Álvarez Pérez, P. (2012b). Los planes institucionales de tutoría y el desarrollo de competencias en el marco del espacio europeo de educación superior: Un estudio desde la perspectiva del alumnado. Perfiles Educativos, 34(137), 28-45. Recuperado de http://www. scopus.com/inward/record.url?eid=2-s2.0-84863856575\&partnerlD=tZOtx3y1

Álvarez, P., López, D., \& Pérez, D. (2016). Programa de tutoría universitaria formativa y desarrollo del proyecto personal del alumnado. Revista de Pedagogía, 37(100), 67-89.

Campoy, T., \& Pantoja, A. (2000). La orientación en la Universidad de Jaén. Revista Española de Orientación y psicopedagogía (REOP), 11(19), 77-106.

Cano, R. \& Paula, A. (2008). Programa Orienta: Plan de acción tutorial universitaria para estudiantes de primer curso. Contextos Educativos, 11(1), 161-179

Cano, R. (2009). Tutoría universitaria y aprendizaje por competencias. ¿Cómo lograrlo? Revista Electrónica Interuniversitaria de Formación del Profesorado, 12(1), 181-204.

Da Re, L., Álvarez, P. R., \& Clerici, R. (2015). Adaptación al contexto universitario italiano del modelo de tutoría formativa para la prevención del abandono y la mejora del rendimiento académico. In Investigar con y para la sociedad (Vol. 2, pp. 721-730). Cádiz: Asociación Interuniversitaria de Investigación Pedagógica -AIDIPE-, 2015. Recuperado de http://dialnet.unirioja.es/servlet/extart?codigo=5188703

Echeverría, B., \& Martínez Clares, P. (2015). Luces entre sombras de la Orientación. Revista Electrónica Interuniversitaria de Formación del Profesorado, 18(2), 1-13.

Esteban, M., Bernardo, A.B., \& Rodríguez-Muñiz, L.J. (2016). Permanencia en la universidad: la importancia de un buen comienzo. Aula Abierta, 44, 1-6. Recuperado de http://www.elsevier.es/es-revista-aula-abierta-389-linkresolver-permanencia-universid ad-importancia-un-buen-S0210277315000153

García, M., Carpintero, E., Biencinto, C., \& Núñez, M. C. (2014). La evaluación del proyecto SOU-estuTUtor: percepción de los mentores como punto de referencia para la mejora de un programa de mentoría universitaria para estudiantes de primero. Revista Complutense de Educación, 25(2), 433-455. Recuperado de http://dialnet. unirioja.es/servlet/articulo?codigo $=4814210$ \&orden $=1$ \&info=link

Gastón, L., \& Rekalde, I. (2016). La tutoría universitaria. El caso del Grado de Pedagogía de la UPV-EHU. Revista d'Innovació Docent Universitària, 8(1), 121-141 Recuperado de http://revistes.ub.edu/index.php/RIDU

Gil-Albarova, A., Martínez, A., Tunnicliffe, A., \& Miguel, J. (2013). Estudiantes universita- 
Tutoría en la Universidad: un estudio de caso en la Facultad de Educación de la Universidad de Murcia

Francisco Javier Pérez Cusó, Cristina González lorente, Natalia González Morga y Mirian MARTÍNEZ JuÁreZ

rios y calidad del Plan de acción tutorial. Valoraciones y mejoras. Revista de Docencia Universitaria, 11(2), 63-87.

González, I., \& León, C. (2014). Diseño de un plan de acción tutorial universitaria para estudiantes de títulos a extinguir: El caso de Psicopedagogía. Revista Española de Orientación y Psicopedagogía, 25(1), 94-100.

González, M., Álvarez, P., Cabrera, L., \& Bethencourt, J. (2007). El abandono de los estudios universitarios: factores determinantes y medidas preventivas. Revista Española de Pedagogía, 71-86.

Haya, I., Calvo, A., \& Rodríguez-Hoyos, C. (2013). La dimensión personal de la tutoría universitaria. Una investigación cualitativa en la Universidad de Cantabria (España). Revista Española de Orientación Y Psicopedagogía, 24(3), 98-113. Recuperado de http://www.scopus.com/inward/record.url?eid=2-s2.084892389830\&part nerID=tZOtx3y1

Lobato, C., \& Guerra, N. (2014). Las tutorías universitarias en el contexto europeo. Orientación y sociedad, 14(1), 1-22. Recuperado de http://www.scielo.org.ar/ scielo. php?script=sci_arttext\&pid=S1851-88932014000100003

Lobato, C., \& Guerra, N. (2016). La tutoría en la educación superior en Iberoamérica: Avances y desafíos, Revista Educar, 52(2), 379-398.

López Gómez, E. (2015). La tutoría en el EEES: propuesta, validación y valoración de un modelo integral (Tesis doctoral). Recuperado de http://e-spacio.uned.es/fez/view/ tesisuned:Educacion-Elopez

Martínez, P., Martínez, M., \& Pérez, J. (2014). Tutoría Universitaria: entorno emergente en la Universidad Europea. Un estudio en la Facultad de Educación de la Universidad de Murcia. Revista de Investigación Educativa, 32(1), 111-138. doi: 10.6018/ rie.32.1.148411

Martínez, P., Pérez, J., \& Martínez, M. (2014). Una (re) visión de la tutoría universitaria: la percepción de estudiantes y tutores de estudios de Grado. Revista de docencia universitaria, 12(1), 269-305.

Martínez, P., Pérez, J., \& Martínez, M. (2016). Las TICS y el entorno virtual para la tutoría universitaria. Educación XX1, 19(1), 287-310. doi:10.5944/educXX1.13942

Martínez, P., Pérez, J., \& Martínez, M. (En prensa).Aplicación de los modelos de gestión de calidad a la tutoría universitaria. Revista Complutense de Educación.

Pérez, J., Martínez, P., \& Martínez, M. (2015). Satisfacción del estudiante universitario con la tutoría. Diseño y validación de un instrumento de medida. Estudios sobre Educación, 29, 81-101.

Rodríguez Espinar, S. (2001). La calidad en la enseñanza universitaria. Ágora Digital, 2, 1-16.

Sobrado, L. (2008). Plan de acción tutorial en los centros docentes universitarios: el rol del profesor tutor. Revista Interuniversitaria de Formación del Profesorado, 22(1), 89108.

Solaguren-Beascoa, M., \& Moreno, L. (2016). Escala de actitudes de los estudiantes universitarios hacia las tutorías académicas. Educación XX1, 19(1), 247-266. doi: 10.5944/ educXX1.14479

Torrecilla, E. M., Rodríguez, M. J., Herrera, M. E., \& Martín, J. F. (2013). Evaluación de ca- 
Tutoría en la Universidad: un estudio de caso en la Facultad de Educación de la Universidad de Murcia

Francisco Javier Pérez Cusó, Cristina González lorente, Natalia González Morga y Mirian MARTíNEZ JuÁreZ

lidad de un proceso de tutoría de titulación universitaria: La perspectiva del estudiante de nuevo ingreso en educación. Revista Española de Orientación Y Psicopedagogía, 24(2), 79-99. Recuperado de http://www.scopus.com/inward/ record.url?eid=2-s2.0 84882967416\&partnerID=tZOtx3y1 